\title{
Prévention de la transmission mère-enfant du VIH : un protocole simple, d'une efficacité remarquable
}

Marc Lallemant, Gonzague Jourdain, Sophie Le Cœur, Nicole Ngo-Giang-Huong, Vallop Thaineua

$>$ En l'absence de prévention, environ $35 \%$ des enfants nés de mère séropositive sont eux-mêmes infectés: $10 \%$ pendant la grossesse, $15 \%$ pendant le travail et $10 \%$ pendant l'allaitement maternel. En 2003, plus de 600000 nouveau-nés ont été infectés par le VIH. Pourtant la majorité de ces infections auraient pu être évitées. En effet, en 1994, l'essai clinique PACTG 076ANRS 024 démontrait l'efficacité remarquable d'un médicament antirétroviral, la zidovudine (AZT) pour prévenir la transmission mère-enfant du VIH au cours de la grossesse et lors de l'accouchement: chez ces femmes qui n'avaient pas allaité, la transmission à l'enfant était de $8 \%$ dans le groupe recevant de la zidovudine et de $23 \%$ dans le groupe placebo, soit une diminution de près de $70 \%$ du risque de transmission [1].

Actuellement, dans la majorité des pays industrialisés où l'allaitement artificiel est la règle pour les mères infectées, la transmission est encore plus faible, inférieure à $2 \%$, grâce à l'utilisation de combinaisons d'antirétroviraux pendant la grossesse et la pratique fréquente de césariennes avant le début du travail. En revanche, dans les pays en voie de développement où vivent la grande majorité des femmes infectées, les programmes de prévention restent encore limités en raison des difficultés rencontrées par les systèmes de santé pour les mettre en œuvre. Dans le cadre d'un programme international de recherche clinique entre la Thaïlande, la France et les États-Unis, les chercheurs du groupe IRD-PHPT (Institut de recherche pour le développement Perinatal HIV prevention trial) ont réalisé, entre 2001 et 2003 en Thaïlande, un essai clinique de phase III, randomisé, en double insu, visant à déterminer si l'adjonction au traitement standard par zidovudine d'une dose de névirapine chez la mère au moment de l'accouchement, et chez son enfant peu après la naissance, pouvait réduire la transmission survenant pendant l'accouchement. Cet essai était financé par le National Institute of Health et l'Agence nationale de recherches sur le sida.

Dans 37 hôpitaux thaïlandais, 1844 femmes enceintes infectées par le VIH ont participé à cet essai. Toutes recevaient un traitement par zidovudine débutant à 28 semaines de grossesse ou le plus tôt possible par la suite, ainsi que leurs enfants pendant la première semaine de vie. De plus, elles n'allaitaient pas leurs enfants. Les femmes ayant donné leur consentement étaient affectées au hasard à l'un des trois groupes du protocole. Les patientes du premier groupe (référence) ne recevaient qu'un traitement standard par zidovudine. Dans le second groupe, les mères recevaient, outre le traitement standard par zidovudine, une dose unique de névirapine au moment de l'accouchement. Dans le troisième groupe, les mères étaient traitées comme celles du deuxième groupe et les enfants recevaient une dose de névirapine 48 à 72 heures après la naissance. Le diagnostic d'infection de l'en-
M. Lallemant: Institut de

recherche pour le

développement, Paris,

France et Department of

Immunology and infectious

diseases, Harvard School of

Public Health, Boston,

États-Unis.

G. Jourdain,

N. Ngo-Giang-Huong:

Department of Immunology

and infectious diseases,

Harvard School of Public

Health, Boston, États-Unis.

S. Le Cœur: Institut national

d'études démographiques,

Paris, France.

V. Thaineua: Ministère de la

Santé publique, Bangkok,

Thaillande.

marc@phpt.org

fant était fait par un test d'amplification génique du VIH (polymerase chain reaction).

$\varepsilon$ n mai 2002, après la première analyse intermédiaire, l'inclusion dans le groupe ne recevant pas de névirapine était interrompue car la transmission dans ce groupe était très supérieure à celle observée dans le groupe où mère et enfant recevaient une dose de névirapine $(6,3 \%$ contre $1,1 \%, p<0,001)$. L'inclusion dans les deux autres groupes de l'étude s'est poursuivie pour déterminer s'il était nécessaire ou non de donner de la névirapine à l'enfant en plus de la dose administrée à la mère. Lors de I'analyse finale, la transmission était de $2,0 \%$ dans le groupe où mères et enfants avaient reçu de la névirapine, et de $2,8 \%$ dans le groupe où seules les mères en avaient reçu (test de non-infériorité: $p=0,03$ ).

Cet essai a donc démontré qu'il était possible, avec un traitement très simple, de réduire le risque de transmission du 
VIH de la mère à l'enfant à un niveau similaire à ceux obtenus avec les procédures complexes utilisées dans les pays industrialisés.

Dans le cadre de cet essai, il était proposé aux femmes immunodéprimées (compte CD $4<250$ cellules $/ \mathrm{mm}^{3}$ ) de démarrer une triple thérapie, prise en charge par le Ministère de la santé, après l'accouchement si ce traitement n'avait pas été institué auparavant.

L'étude de 269 femmes immunodéprimées (48 n'ayant pas reçu de névirapine au moment de l'accouchement et 221 en ayant reçu) a montré qu'après six mois de traitement, l'augmentation des CD4 était similaire dans les deux groupes. En revanche, la proportion de patientes ayant une charge virale inférieure à 50 copies $/ \mathrm{ml}$ était de $68 \%$ chez les patientes ayant reçu de la névirapine au moment de l'accouchement, et de $49 \%$ chez les autres $(p=0,03)$. Dix jours après l'accouchement, aucune mutation du VIH associée à la résistance à la névirapine n'était trouvée chez les femmes n'ayant pas reçu de névirapine au moment de l'accouchement, alors qu'au moins une mutation était trouvée chez $32 \%$ des femmes en ayant reçu (K103N et G190A, essentiellement, diagnostiquées par les techniques de séquençage classiques). Parmi les femmes qui avaient reçu de la névirapine au moment de l'accouchement et qui présentaient des virus mutants, $38 \%$ avaient une charge virale inférieure à 50 copies/ml après 6 mois de traitement, contre $52 \%$ chez celles chez qui des virus mutants n'avaient pas étés retrouvés $(p=0,08)[2]$.

En décembre 2003, après avoir examiné l'ensemble de ces résultats, le Ministère de la santé de Thaïlande a décidé de recommander la prophylaxie par zidovudine et dose unique de névirapine pour son programme national de prévention de la transmission mère-enfant du VIH, tout en préconisant de commencer une triple thérapie en cours de grossesse en cas d'indication thérapeutique pour la mère $\left(C D 4<200 / \mathrm{mm}^{3}\right)$. En fait, ces femmes qui ont besoin de débuter une thérapie pour leur propre santé sont, du fait de leur immunosuppression et de leur propension à avoir une charge virale élevée, les femmes chez lesquelles le développement des résistances après une simple exposition à la névirapine est la plus probable. L'Organisation mondiale de la santé a adopté cette stratégie lors de la révision de ses recommandations au début de 2004 [3].

L'utilisation de la zidovudine et d'une dose unique de névirapine s'est révélée d'une grande simplicité et d'une exceptionnelle efficacité pour la prévention de la transmission du VIH de la mère à l'enfant. Elle ne doit pas compromettre les chances de succès thérapeutique chez les mères qui y ont eu recours. Des essais sont déjà en cours qui laissent espérer que l'administration d'une combinaison antirétrovirale à la mère pendant les quelques semaines qui suivent l'exposition à la dose unique de névirapine peut prévenir la sélection des mutations de résistance [4]. $\diamond$

Prevention of mother-to-child transmission of HIV:

a simple and highly efficacious regimen

\section{REMERCIEMENTS}

Les obstétriciens, pédiatres, infirmières, conseillers, techniciens de laboratoire et personnels administratifs des 37 hôpitaux qui ont participé à l'étude ainsi que tout le personnel de PHPT.

Les institutions qui ont soutenu PHPT sont: en Thaïlande: le Ministère de la Santé publique, les universités de Chiang Mai, de Khon Kaen et de Mahidol; en France: l'unité de recherche 054 de l'Institut de recherche pour le développement (IRD), l'Institut national de la santé et de la recherche médicale (Inserm) Erm 0321 et l'Institut national d'études démographiques (INED); aux États-Unis: l'Harvard School of Public Health, Department of Immunology and Infectious Diseases, l'University of Massachusetts, le National Institute of Child Health and Human Development (NICHD), le Fogarty International Center du National Institutes of Health (NIH).

La recherche a été financée par le NICHD du National Institutes of Health (RO1-HD 39615), l'Agence Nationale de Recherches sur le Sida (ANRS 1208), l'IRD, l'INED, le Ministère français des Affaires étrangères, le Ministry of Public Health et le Department of Technical and Economic Cooperation, en Thaïlande. Les produits pour étude ont été fournis par les laboratoires Boehringer-Ingelheim et GlaxoSmith-Kline, et les réactifs pour les tests de PCR-ADN-VIH par le laboratoire Roche Molecular Systems.

\section{RÉFÉRENCES}

1. Sperling RS, Shapiro DE, Coombs RW, et al. Maternal viral load, zidovudine treatment, and the risk of transmission of human immunodeficiency virus type 1 from mother to infant. Pediatric AIDS Clinical Trials Group Protocol 076 Study Group. N Engl J Med 1996; 335: 1621-9.

2. Jourdain G, Ngo-Giang-Huong N, Le Coeur S, et al. Intrapartum exposure to nevirapine and subsequent maternal responses to nevirapine-based antiretroviral therapy. N Engl I Med 2004; 351: 229-40.

3. World Health Organization. Antiretroviral drugs for treating pregnant women and preventing HIV infection in infants. Guidelines on care, treatment and support for women living with HIV/AIDS and their children in resource-constrained settings. Genève: 0MS, 2004: $50 \mathrm{p}$.

http://www.who.int/3by5/arv_pmtct/en/.

4. Mclntyre J, Martinson N and Investigators for the Trial 1413. Addition of short course combivir (CBV) to single dose viramune (sdNVP) for prevention of mother-to-child transmission (MTCT) of HIV-1 can significantly decrease the subsequent development of maternal NNRT-resistant virus. Bangkok: $X V$ Conférence Internationale sur le Sida, juillet 2004, LbOrB09. 